\section{AB0620 HIGH RISK OF MISTAKEN CLASSIFICATION OF PRIMARY ANTIPHOSPHOLIPID SYNDROME AS SYSTEMIC LUPUS ERYTHEMATOSUSACCORDING TO THE SLICC CRITERIA: ANALYSIS OF A COHORT OF 214 ANTIPHOSPHOLIPID PATIENTS}

R. Paule, N. Costedoat-Chalumeau, N. MOREL, V. LE GUERN, M. Fredi, L. Coutte, M. Belhocine, L. Mouthon, C. Le Jeunne, A. Chauvin, J.-C. Piette. APHP, PARIS, France

Background: The diagnosis of systemic lupus erythematosus (SLE) is based on the association of clinical and biological manifestations and on clinical experience. In 2012, a major revision by the Systemic Lupus International Collaborating Clinics (SLICC) group sought to improve their sensitivity and specificity. In replications, the SLICC classification produced fewer errors than the previous version its higher sensitivity but lower specificity meant that some patients could be classified with SLE although they had another disease. In fact, the distinction between PAPS, APS associated with SLE, and isolated SLE may be difficult in some cases because the two diseases share some clinical and biological manifestations.

Objectives: To assess the limitations of the SLICC (Systemic Lupus International Collaborating Clinics) classification criteria for systemic lupus erythematosus (SLE), in patients with primary antiphospholipid syndrome (PAPS).

Methods: Retrospective study of a cohort of APS patients (Sydney criteria). We successively excluded patients with ${ }^{1}$ at least one "SLE-specific" manifestation (biopsy-proven SLE nephropathy, arthritis, cutaneous, or neurologic SLE manifestations, pericarditis, autoimmune haemolytic anaemia, oral and nasal ulcers, non-scarring alopecia, anti-dsDNA, and anti-Sm antibodies), ${ }^{2}$ any other autoimmune connective tissue disease, and/or ${ }^{3}$ antinuclear antibodies $>1 / 320$. Careful file review confirmed PAPS among the remaining patients. We then assessed the number of SLICC criteria each patient met.

Results: Among these 214 APS patients, we excluded 85 with at least one SLEspecific manifestation, 8 with another connective tissue disease, and 21 with antinuclear antibodies $>1 / 320$, leaving 100 patients with primary APS. Among them, $28 \%$ met at least 4 SLICC classification criteria including one clinical and one immunological criterion (antiphospholipid antibodies, aPL, by definition) and could thus theoretically be classified with SLE. Fourteen had an arterial phenotype $(50 \%), 9$ a history of catastrophic APS $(32 \%)$, and 18 a triple-positive profile for aPL (64\%). None had developed SLE during a median follow-up of 12 [6.5-17] years.

Conclusions: Because $28 \%$ of our patients with longstanding and strictly defined PAPS could be mistakenly classified as SLE, they were at risk of deleterious therapeutic management. We therefore suggest that any future classification for SLE should specifically require at least one SLE-specific criterion for patients with aPL.

\section{REFERENCES:}

[1] Petri M, Orbai A-M, Alarcón GS, et al. Derivation and validation of the Systemic Lupus International Collaborating Clinics classification criteria for systemic lupus erythematosus. Arthritis Rheum 2012;64:2677-86. doi:10.1002/art.34473

[2] Piette JC, Wechsler B, Frances C, et al. Exclusion criteria for primary antiphospholipid syndrome. J Rheumato/1993;20:1802-4.

[3] Asherson RA, Khamashta MA, Ordi-Ros J, et al. The 'primary' antiphospholipid syndrome: major clinical and serological features. Medicine (Baltimore) 1989;68:366-74.

[4] Gómez-Puerta JA, Martín H, Amigo M-C, et al. Long-term follow-up in 128 patients with primary antiphospholipid syndrome: do they develop lupus? Medicine (Baltimore) 2005;84:225-30.

Disclosure of Interest: None declared DOI: 10.1136/annrheumdis-2018-eular.3207

\section{AB0621 EPIDEMIOLOGY, CLINICAL CHARACTERISTICS AND THERAPY APPORACHES OF A RETROSPECTIVE COHORT OF PAEDIATRIC SYSTEMIC LUPUS ERYTHEMATOSUS IN A TERTIARY CENTRE}

R.M. Alcobendas, S.M. Loza, A.R. Camba, C.U. Gascon. Pediatric Rheumatology, university hospital La Paz, Madrid, Spain

Background: Systemic lupus erythematosus (SLE) is a chronic autoimmune dis ease, potentially severe, with broad clinical spectrum, which can affect multiple organs and systems.

Objectives: To analyse the initial manifestations, laboratory examinations and therapeutic approaches of patients diagnosed with childhood onset systemic lupus erythematosus (c-SLE) followed in a tertiary hospital in the last 14 years. Methods: Retrospective chart review. Inclusion criteria were: children under 18 years diagnosed with c-SLE between January 2003 and January 2017.
Results: During the study period, 38 patients were identified (ratio female/male: $3 / 1$ ). The mean age at the onset of disease was 11.5 years (range $6-17$ ). All had caucasic origin, except 5 coming from South America, 2 from India and 1 from Africa. Onset of the disease took place in spring in 22 (58\%) patients, while in 10 $(26 \%)$ patients onset was in summer, $5(13 \%)$ in autumn and only one (3\%) in winter season.

The most frequently clinical manifestations found at the debut were cutaneous involvement $(66 \%$, predominantly in the form of a malar rash), renal $(65 \%)$ and joint $(60 \%, 48 \%$ arthralgia and $52 \%$ polyarthritis). Other manifestations were fever $(50 \%)$, cytopenias $(39 \%)$, asthenia $(36 \%)$, serositis and neurological clinic (both $26 \%$ ) and oral aphthosis (23\%). Among the neurological manifestations, 4 patients showed bradypsychia, 3 headache, 2 seizures and 1 manifested a paralysis of the sixth cranial nerve. Only 1 patient presented macrophage activation syndrome after primoinfection by Ebstein Barr Virus. During the study period, no deaths ocurred.

Ten patients also had some other associated autoimmune disease: 6 hypothyroid isms, 2 lgA deficiency, 1 vitiligo and 1 celiac disease. No case of diabetes mellitus was identified.

Regarding immunology, in all cases, positive antinuclear antibodies (ANA) were detected, although with variable titers $(1 / 80-1 / 5120)$. Of the 25 patients who presented renal disease at the onset, 19 associated Anti-dsDNA antibodies positive at the initial time of the determination. Although antibodies related to antiphospholipid syndrome (APS) (anticardiolipin, anti-2glycoprotein and lupus anticoagulant) were detected in 12 patients (32\%), only two developed associated clinical manifestations (both deep vein thrombosis in the lower limbs).

Regarding treatment, all patients required corticosteroids. Therapy with acetylsalicylic acid was indicated in all patients with APS-associated immunology. Only 6 patients received treatment with corticosteroids and hydroxychloroquine exclusively. Nineteen (50\%) patients initially received azathioprine therapy, being necessary to switch to mycophenolate mofetil for lack of response in eleven, receiving the last treatment 20 patients finally (52\%). Eighteen patients $(47 \%)$ received cyclophosphamide therapy, 16 of them as a consequence of their renal involvement. In addition, biologic therapy (rituximab and belimumab, respectively) was used in two multirefractory patients.

Conclusions: As widely already reported, SLE is a disease that affects predominantly women. Moreover, as it has been previously described in the literature the most frequently initial manifestations found in c-SLE are cutaneous, renal and articular. However, a large variability of onset symptoms exists, thus c-SLE should be ruled out in patients with multisystemic involvement

Disclosure of Interest: None declared

DOI: 10.1136/annrheumdis-2018-eular.5480

\section{AB0622 CHARACTERISTICS OF SYSTEMIC LUPUS ERYTHEMATOSUS PATIENTS IN MALTA; A POPULATION BASED CROSS-SECTIONAL COHORT STUDY}

\section{R. Magro, A.A. Borg. Rheumatology, Mater Dei Hospital, Msida, Malta}

Background: Systemic Lupus Erythematosus (SLE) is an autoimmune disorder that involves multiple systems including the skin, musculoskeletal, renal, neuro logical, haematologic, cardiovascular and respiratory systems.

Objectives: The aim of this study was to characterise the patients with systemic lupus erythematosus living in Malta, in terms of age of disease onset, BMI, co morbidities, drug history, disease activity, damage and other factors including fatigue, sleep quality, depression, anxiety and vitamin D level.

Methods: The study consisted of a cross-sectional cohort study of all known SLE patients, over the age of 18, living in Malta. 92 patients who fulfilled the SLICC classification criteria for SLE, gave informed consent and an interview was carried out. Fatigue, anxiety, depression, sleep quality and disability were assessed respectively by filling in the following questionnaires: Fatigue Severity Scale (FSS), Hospital Anxiety and Depression Scale (HADS), Pittsburgh Sleep Quality Index (PSQI) and modified Health Assessment Questionnaire (mHAQ).

Results: $92.4 \%$ of SLE patients studied were female. Table 1 summarises the characteristics of the SLE patients.

$23.9 \%$ of SLE patients were in remission (SLEDAI-2K 0), while $52.2 \%$ had a low disease activity (SLEDAI-2K 1-5) at the time of the interview. 20.7\% and $3.3 \%$ had a moderate (SLEDAI-2K 6-10) and high (SLEDAI-2K 11-19) disease activity respectively. A significant positive correlation was noted between function meas ured by $\mathrm{mHAQ}$ and SLEDAI ( $\mathrm{R}=0.417, \mathrm{p}=0.000) .56 .5 \%$ were noted to have an abnormally high level of fatigue (FSS $>3.7$ ). $6.5 \%$ were noted to have depression (HADS D>10) and $35.9 \%$ had anxiety (HADS A>10). 55.4\% were noted to have poor sleep quality (PSQI $>5$ ) and $26.1 \%$ had an abnormal level of function ( $\mathrm{mHAQ}$ $>0.3$ ). $15.2 \%$ were found to have vitamin $D$ deficiency and $27.2 \%$ were vitamin $D$ insufficient. 